\title{
Optimal Data Delivery in Wireless Sensor Networks in the Energy and Latency Domains
}

\author{
M. Borghini, F. Cuomo, \\ T. Melodia, U. Monaco \\ Univ. of Rome "La Sapienza" \\ \{lastname\}@infocom.uniroma1.it
}

\author{
F. Ricciato \\ Forschungszentrum \\ Telekommunikation Wien (ftw.) \\ ricciato@ftw.at
}

\begin{abstract}
In this paper we address the problem of optimal data gathering in wireless sensor networks (WSN). The goal of this work is to develop algorithms and techniques in order to minimize the data delivery latency and at the same time balance the energy consumption among the nodes, so as to maximize the network lifetime. Following an incrementalcomplexity approach, several mathematical programming problems are proposed with focus on different network aspects. First, the static routing problem is formulated for large and dense WSNs. Then, an accurate network model is proposed that captures the tradeoff between the data delivery latency and the network energy consumption by modeling the interactions among the routing, medium access control and physical layers. Finally, we consider dynamic rerouting and scheduling. For each problem we provide extensive simulations results for reference scenarios.

The proposed models provide a deeper insight into the problem of timely and energy efficient data gathering. Along with the simulation results reported here they provide useful guidelines for the design of effective WSNs.
\end{abstract}

\section{Introduction}

Wireless sensor networks [1] are composed of small miniaturized devices endowed with limited sensing, processing and computational capabilities. Wireless sensors can be densely deployed across the monitored area, and enable a broad range of applications in different fields like environmental monitoring, surveillance, law enforcement, etc.

In the typical applications the sensors monitor their neighboring area, extract information and send sensed data to remote sinks that reconstruct the overall characteristics of the phenomenon [1]. Since wireless sensors are typically low-cost, low-power and short-range devices, multi-hop routes are used to relay data from the mon- itored area to the sink. These paths are typically built on-demand (reactive routing) or dynamically pre-computed (proactive routing) [2]. In the former case, path computation is triggered by the occurrence of specific events or upon request from the application; in the latter, the routes are determined before they are really used. The aggregate of multi-hop paths used to relay data to the sink can be seen as a data-delivery tree.

In this context, data aggregation has been proposed as an essential paradigm for routing in WSNs [3]. The idea is to combine the data coming from different sources enroute, thus eliminating redundancy, minimizing the number of transmissions and saving energy. This relies on the assumption, true in many practical cases, that data sensed by different sensors are to some extent spatially correlated [4]. This paradigm shifts the focus from the traditional addresscentric approaches (finding short routes between pairs of addressable end-nodes) to the so-called data-centric approach (finding routes from multiple sources to a single destination and allowing in-network consolidation of redundant data). For this reason, data-delivery trees are also referred to as data-aggregation trees (DA-trees).

Intuitively, due to the dense sensor deployment, many different DA-trees can be used to relay data from the event area to the sink, which greatly affects many key performance metrics of the WSN, such as network lifetime, energy consumption, network availability and real-time applications support. Moreover, the characteristics of DA-trees may differ in many domains, in primis with respect to energy consumption, i.e., the energy needed to gather sensed data to the sink, and also in the latency domain, i.e., the time needed to deliver data to the sink. According to application requirements, energy or latency oriented design approaches should be pursued.

Previous works considered the problem of minimizing the energy consumption of a WSN. Chang and Tassiulas [5],[6] formulate the maximum lifetime routing problem for a WSN as an integer linear program, and propose heuristic algorithms to determine approximate solutions. In [7], 
a distributed algorithm is presented to determine the maximum lifetime, based on the Garg-Koenemann [8] algorithm for multi-commodity flows. In [9], the authors formulate the maximum lifetime routing problem as a maximum concurrent flows problem, and propose a distributed routing algorithm that finds the optimal solution within an asymptotically small relative error, hence giving lower bounds on its performance. In [10], a heuristic near-optimal solution is proposed for the problem of maximum lifetime routing that can be computed in polynomial time. In [11], the authors explore the fundamental bounds of WSN lifetime, that allow calibrating the performance of collaborative strategies.

In general, in the above papers, the routing issue is dealt with from an energy consumption standpoint. Conversely, we extend the analysis to the latency domain. This allows us to investigate energy-latency trade offs for optimal data delivery in WSNs. This problem has been previously investigated in [12]. There, the focus is on minimizing the energy consumption given a time constraint, by leveraging the modulation scaling technique. However, the problem of finding a minimum latency data-delivery tree is not investigated.

The objective of this paper is to provide a wide-ranging analysis of the impact of different network design strategies for data gathering. To this aim, we define several optimization problems in the energy-latency domain and tackle them with a multi-target approach. The mathematical framework proposed here allows the WSN designer to foresee the impact of different design choices on optimal DA-trees as a function of different performance targets. The presented results will help the researchers to gain a deeper understanding of the fundamental characteristics of WSNs in the energy-latency domains.

The following of the paper is organized as follows. Section 2 introduces the general network model. Section 3 presents a formulation of the optimal data gathering with static routing problem, while a different model to investigate the problem of re-routing is presented in Section 4. In Section 5, we draw the main conclusions and outline future work.

\section{Network model}

We consider a scenario where wireless sensors are randomly deployed in a bi-dimensional terrain. The network of sensors is modelled as a graph whose vertexes (sensors) are represented by the set $\mathcal{N}$, with $N=|\mathcal{N}|$. A subset of the sensors $\mathcal{S} \subseteq \mathcal{N}$ (in the following referred to as sources) generates information to be relayed to a sink (in the following labeled by $O$ ). We denote by $d_{i j}$ the Euclidean distance between sensors $i$ and $j$, while $d_{i}$ denotes the distance between sensor $i$ and the sink. Each sensor is characterized by a maximum transmission range $T_{R}$ that accordingly defines the set $C_{i}$ of neighbors for the sensor $i$. The neighbors of a sensor are the candidate next hops towards the sink. We assume that transmitting a unit of information from sensor $i$ to sensor $j$ requires a power proportional to $d^{\alpha}$ with $2 \leq \alpha \leq 5$. In the following, unless otherwise specified, we assume free space attenuation $\alpha=2$.

\section{Optimal data gathering with static routing}

In this section we analyze optimal data-delivery trees in dense network scenarios with static routing, i.e., data gathering paths do not change with time. The objective of this analysis is to investigate the basic interactions between routing and energy consumption by means of global optimization methods.

\subsection{Problem formulation}

The problem of optimal data gathering in WSNs can be formulated as a classical network flow transportation problem [13]. However, a wide subset of such problems has been proven to be NP-complete [14]. Hence, in order to analyze large scale networks, we rely on a set of simplifying assumptions:

- sensors can arbitrarily split the traffic that they generate/relay and accordingly transmit it to several different neighbors (multi-path routing). This results in the linear relaxation of the routing variables, also referred to as "splittable traffic" [15];

- sensors do not perform data aggregation;

- sensors communicate through an ideal shared medium, i.e., we do not model contention at the MAC layer or finite transmission power at the physical layer.

Moreover, without loss of generality, we assume a continuous-time transmission of information, so that we can define the following quantities:

- $g_{i}$ is the information flow generated by sensor $i$ and destined to the sink;

- $|\mathcal{S}|=N$, i.e., all sensors generate the same amount of information to be sent to the sink, i.e., $g_{i}=g, \quad \forall i \in$ $\mathcal{N}$

- $q(i, j)$ is the information flow transmitted ${ }^{1}$ from sensor $i$ to sensor $j$;

- $E_{i j}=\beta d_{i j}^{\alpha} \cdot q(i, j)$ is the energy needed to transmit the flow $q(i, j)$ over a distance $d_{i j}$ where $\beta$ is a constant $\left[\right.$ Joule $/\left(\right.$ bits $\left.\left.\cdot m^{\alpha}\right)\right]$;

1 Note the different usage of terms transmitted and generated. 
- $E_{i}=\sum_{j \in C_{i}} E_{i j}$ is the total energy consumption at sensor $i$; note that this energy consumption model only takes into account the radiated power.

With such positions, the objective of minimizing and/or balancing the network energy consumption can be achieved by optimally controlling the fraction of information (therefore, of energy) transmitted by each sensor to each of its neighbors, which defines the multi-path routing strategy for the whole network. Hence the optimization problem consists of finding the set of variables $q(i, j)$ (for all sensor pairs $(i, j)$ ) that:

- minimizes the maximum energy consumption for a single sensor $E_{\text {max }}=\max _{i}\left\{E_{i}\right\}$, named $\operatorname{ROM}_{E}$ (Routing Optimization Maximum Energy);

- minimizes the total energy consumption of the network $E_{t o t}=\sum_{i=1, N} E_{i}$, named ROT ${ }_{E}$ (Routing Optimization Total Energy).

Since minimizing the total energy consumption $E_{t o t}$ is equivalent to minimizing the mean energy $E_{\text {mean }}=$ $\frac{1}{N} E_{t o t}$, we provide a Linear Programming (LP) formulation for the general case of mixed optimization, where the objective function to be minimized is a linear combination of $E_{\max }$ and $\frac{1}{N} E_{t o t}$. By tuning the coefficient $\gamma$ one can shift from $\operatorname{ROM}_{E}(\gamma \approx 1)$ to $\operatorname{ROT}_{E}(\gamma \approx 0)$.

$$
\begin{gathered}
\text { Minimize: } \\
\gamma E_{\text {max }}+(1-\gamma) \cdot \frac{1}{N} E_{\text {tot }} \\
\text { subject to: } \\
q(i, j) \geq 0, \quad \forall j \in C_{i}, \forall i \in \mathcal{N} \\
\sum_{k: i \in C_{k}} q(k, i)+g_{i}=\sum_{j \in C_{i}} q(i, j), \quad \forall i \in \mathcal{N}
\end{gathered}
$$

It is to be noticed that, since we do not consider data coding/aggregation, the information flow is additive (constraint (3)). Hence, we do not need to discriminate between information flows originated by different sensors (singlecommodity flow problem).

\subsection{Analysis of the optimal routing patterns}

We implemented the optimization Problem (1) in AMPL [16] and solved it with CPLEX [17]. To determine the main characteristics of the optimal data-delivery trees, we averaged over a statistically significant number of scenarios. We initially set the radio transmission range $T_{R}=d^{\max }=$ $\max _{i}\left\{d_{i}\right\}, \beta=1$ and considered $N=1000$ sensors deployed in a circular terrain with radius $r=d^{\max }=1 \mathrm{~m}$. Figure 1 reports the per-sensor energy consumptions $E_{i}$ for all the sensors as a function of the distance from the sink $d_{i}$ for different values of $\gamma$.

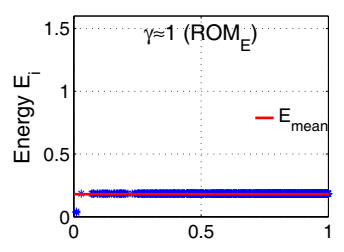

(a)

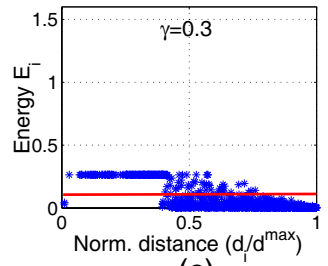

(c)

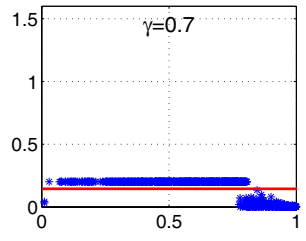

(b)

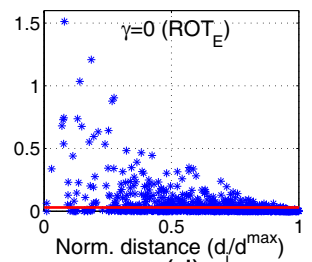

(d)
Figure 1. Per-sensor energy consumption $E_{i}$ versus $d_{i}$ for different optimization criteria.

In the $R O T_{E}$ case, $(\gamma=0$, Fig. 1 (d)) it is known that an extremal solution of the Problem (1) exists [13], with either $q(i, j)=0$ or $q(i, j)=g$ for each $(i, j)$ pair. This solution represents single-path data routing (non-splittable traffic) from each source towards the sink, e.g., each sensor selects only one next hop. Since the energy consumption of a sensor is proportional to $d_{i j}^{\alpha}$, $R O T_{E}$ minimizes the distance between each pair of sensors along the data-gathering paths, e.g., each next hop is close to the corresponding transmitting sensor. Therefore exterior sensors (i.e., sensors far from the sink) only consume energy to transmit the data they generate, while interior ones (i.e., sensors close to the sink) also relay data for other sensors. Hence, the $R O T_{E}$ approach leads to a higher energy consumption $E_{i}$ for sensors closer to the sink.

When $\gamma>0$, a mixed $E_{\max }-E_{t o t}$ optimization is performed, and the optimal data-delivery trees are computed to reduce the maximum energy consumption $E_{\max }$, at the price of a higher $E_{t o t}$. For $\gamma \approx 1\left(R O M_{E}\right.$, Fig. 1(a)), the objective of minimizing the maximum energy consumption implies that almost all sensors consume the same energy $\left(E_{i}=E_{\max } \simeq E_{\text {mean }}\right)$. With respect to the routing derived by the $R O T_{E}$ criterium, $R O M_{E}$ reduces the $E_{\max }$ by a factor 8 , but $E_{\text {tot }}$ is higher by a factor 4 .

To better understand how data flows are routed through the network, we report the normalized energy consumption $E_{i j} / E_{\text {max }}$, for each transmission of $q(i, j)$ bits from sensor $i$ to sensor $j$, for $R O T_{E}$ and $R O M_{E}$ (Fig. 2(a) and 2(b), respectively). As discussed above, in the $R O T_{E}$ case each sensor has one near next hop along the path towards the sink $\left(d_{i} \cong d_{j}\right)$. In particular, we found that $70 \%$ of the times the next hop is the nearest sensor towards the sink at distance $d_{\text {near }}$, and $95 \%$ of the times it is distant up to 


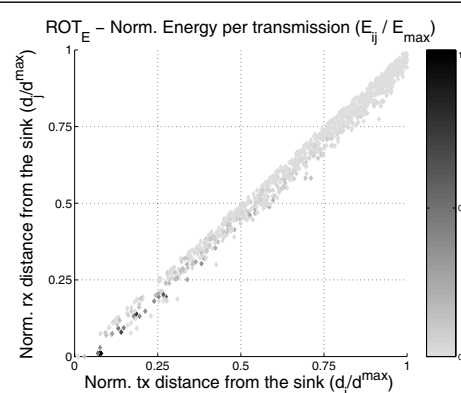

(a) $\operatorname{ROT}_{E}\left(T_{R}=d^{\max }\right)$

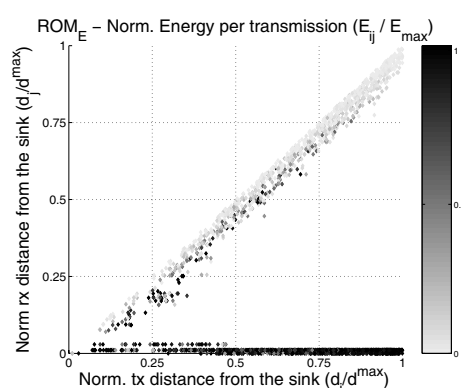

(b) $R O M_{E}\left(T_{R}=d^{\max }\right)$

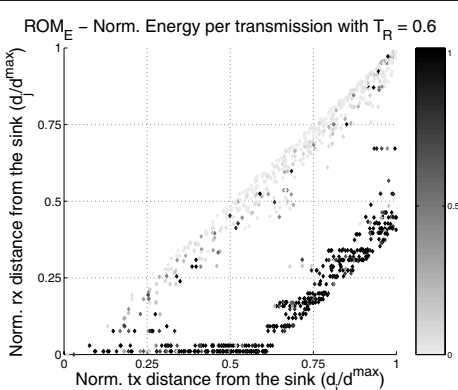

(c) $R O M_{E}\left(T_{R}=0.6 \cdot d^{\max }\right)$

Figure 2. Normalized energy consumption $E_{i j} / E_{\max }$ for the transmission of $q(i, j)$ bit from sensor $i$ (at distance $d_{i}$ from the sink) to sensor $j$ (at distance $d_{j}$ from the sink) in a 1000 sensors network.

$2 \cdot d_{\text {near }}$. As a consequence, sensors closer to the sink need to relay a higher amount of data, thus consuming more energy. Conversely, when $R O M_{E}$ is performed, far transmissions also occur (points with $d_{j} \simeq 0$ in Fig. 2(b)). These transmissions are directed to the sink or to sensors closer to it (namely the far next hops), and are responsible for most of the energy consumption. Load balancing is achieved by means of these far transmissions, i.e., each sensor tries to spend $E_{\max }$ units of energy by routing data over multiple near and far next hops. An interesting point concerns the number of next hops selected by each sensor. In fact, the constraint (2) potentially allows its value to reach $N$. Surprisingly, we found that the number of next hops is always very limited: approximately $6 \%$ of the sensors have 1 next hop, $86 \%$ select 2 next hops and $7 \%$ transmit to 3 next hops (up to 6 in very few cases). In particular, in the case of 2 next hops, transmissions are almost always directed to one near next hop, to which most of the data are transmitted, with low energy consumption; and to one far next hop, to which a smaller amount of data are transmitted, but with most of the energy consumption.

The differences between $R O M_{E}$ and $R O T_{E}$ also have an impact on the network lifetime. By assuming that the network dies when the first sensor finishes its energy, in the $R O T_{E}$ case the network lifetime is highly influenced by the unbalanced use of energy in the sensors. On the contrary, in the $R O M_{E}$ case all sensors deplete their batteries at the same time, which can lead to the extended network lifetime.

We repeated the data-delivery tree analysis with $T_{R}<$ $d^{\max }$. In the $R O T_{E}$ case, until the network is connected, a reduction of the transmission range does not produce significant changes in optimal data-delivery trees, with respect to that obtained with $T_{R}=d^{\max }$, either in terms of number of the next hops (always one) or in terms of the mean distance $d_{i j}$. For the $R O M_{E}$, Fig. 2(c) reports the normalized energy consumption for each transmission when $T_{R}=0.6 \cdot d^{\max }$. The key routing characteristics that we underlined in the case of $T_{R}=d^{\max }$ continue to hold, but the radio range constraint causes an overall higher energy consumption. In fact, as in the $R O T_{E}$ case, exterior sensors that cannot directly transmit to the sink load interior ones that must spend more energy. Hence, until $T_{R}$ is sufficiently high to balance the energy consumption, every sensor consumes the same energy $E_{\text {max }}=E_{\text {mean }}$ (with $E_{\text {max }}$ increasing as $T_{R}$ decreases), while if $T_{R}$ decreases below a "critical range" $\left(0.6 \cdot d^{\max }\right.$ in Fig. 3$)$, the energy consumption cannot be balanced (in this case $E_{\max }>E_{\text {mean }}$ ). For small values of the radio range, $R O M_{E}$ data-delivery trees are similar to the $R O T_{E}$ ones, since every sensor can only transmit data to a near next hop. This behavior explains the rise of the $E_{\text {max }}$ and the decrease of the $E_{\text {mean }}$ i.e., the total energy $E_{\text {tot }}$.

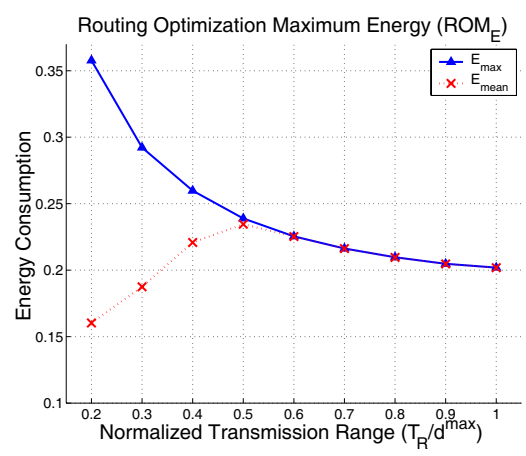

Figure 3. Max and Mean energy consumption as a function of the Normalized Transmission Range $T_{R} / d^{\max }$ for $R O M_{E}$. 


\section{Optimal data gathering with re-routing}

In this section we introduce a more accurate energy model, take into account contention at the MAC layer, and consider data aggregation strategies. The model presented in this section allows analyzing dynamical scenarios, where the optimal DA-trees are determined sequentially for different events, thus taking into account the residual energy at each sensor. Moreover, it captures the interdependencies between the network and medium access control layers, by considering the latency of the data delivery process. We focus on single-path data flows, i.e., the splittable traffic hypothesis does not hold. Hence, we provide an Integer Linear Programming (ILP) formulation. Due to the NPcompleteness of the new problem formulation, in the remainder of the paper only a subset of the sensors generate data.

As for the energy model, consumption per bit at the physical layer can be accurately modeled as:

$$
E^{\text {trans }}=E_{\text {elec }}^{\text {trans }}+\beta d^{\alpha}, E^{r e c}=E_{\text {elec }}^{\text {rec }}
$$

where $E^{\text {trans }}$ and $E^{r e c}$ are the energy consumption at the transmitter and receiver devices of a sensor, respectively. In particular, $E_{\text {elec }}$ is a distance independent term that takes into account overheads of sensor electronics (PLLs, VCOs, bias currents, etc.) and digital processing [Joule/bits]. As in [18], we assume that $E_{\text {elec }}^{\text {trans }}=E_{\text {elec }}^{\text {rec }}=E_{\text {elec }}$. The overall expression for the transmission of one bit on a wireless link simplifies to $2 \cdot E_{\text {elec }}+\beta d^{\alpha}$. We set $2 * E_{\text {elec }}=570 \mathrm{~nJ}$, $\alpha=2$, while $\beta$ is dependent on the maximum transmission range $\left(T_{R}\right)$ of the radio device $\left(\beta=740 n J / T_{R}^{2}\right)$. We further assume that all sensors in $\mathcal{S}$ sense correlated information. Therefore, each sensor produces packets of $g$ bits, and when a relay sensor receives two packets from two different nodes, it can aggregate the two packets and send a single $g$-bits packet towards the sink (e.g., in the case of $\mathrm{min} / \mathrm{max} / \mathrm{mean}$ data aggregation functions). Without loss of generality, we consider $g=1$.

We consider a scenario with multiple events. For each event, the sensors in $\mathcal{S}$ generate information (e.g., a data packet) that has to be delivered to the sink. An optimal DAtree is then calculated for that event. Hence, for each event we calculate a new DA-tree from the sources to sink, on the basis of the variations in the battery level of the involved devices and achieve an optimal strategy for a dynamical scenario. We refer to this procedure as re-routing. Moreover, we model the scheduling of data transmissions on the DAtrees. Transmissions from sensors within reciprocal radio range are scheduled in different time slots. Multiple time slots are hence required to gather the information generated by the sensors in $\mathcal{S}$. We can now introduce the following definitions.
Definition 1 In a multiple events case, the lifetime of the WSN is the maximum number of events that can be observed before at least one sensor in $\mathcal{S}$ loses connectivity to the sink.

Definition 2 The network latency $T_{M A X}$ for an event is the total number of time-slots required to gather the information generated by the sensors in $\mathcal{S}$ for that event, where $\mathcal{T}=\left\{1, \ldots, T_{M A X}\right\}$ is the set of time slots required to deliver the data measured by the sensors in $\mathcal{S}$ to the sink.

Definition 3 The sensing coverage of the network is the portion of the area that is monitored by the sensors, i.e., that is within the sensing range of at least one sensor that has not depleted its battery.

We propose two different formulations of the data delivery problem, namely a latency-oriented and an energyoriented one. The objective of the former is to find, given a sequence of events, the sequence of minimum-latency DA-trees $\left(\mathrm{R}^{2} \mathrm{OB}_{L}-\right.$ Re-Routing optimal balancing, latencyoriented), and the minimum-latency scheduling on those trees. For each event, the minimum-energy DA-tree is then selected among those that yield minimum-latency. Conversely, the objective of the energy-oriented formulation is to find the sequence of energy efficient DA-trees, and then the minimum-latency scheduling on those DA-trees $\left(\mathrm{R}^{2} \mathrm{OB}_{E}\right.$ - Re-Routing optimal balancing, energy-oriented $)$. Algorithms 1 and 2 describe the steps taken to solve both problems.

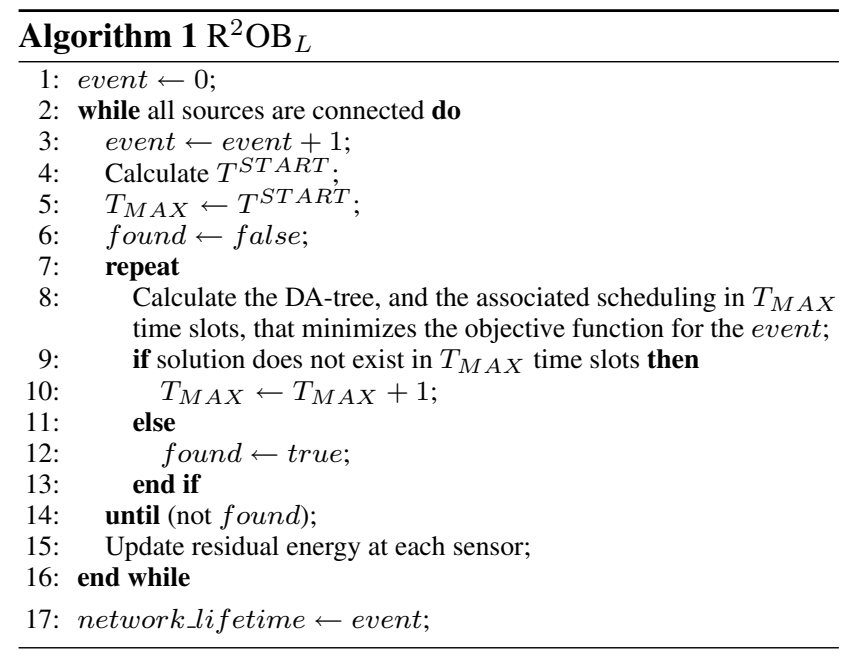

In both algorithms, the latency $T_{M A X}$ is calculated by solving ILPs with increasing latency as input argument (e.g., 3,4,5 time slots). The first instance of the ILP that admits a solution defines the minimum latency for the considered scenario. The value $T_{M A X}$ is initialized to the minimum possible latency for the considered topology, which is equal to

$$
T^{S T A R T}=\max (h, T)
$$




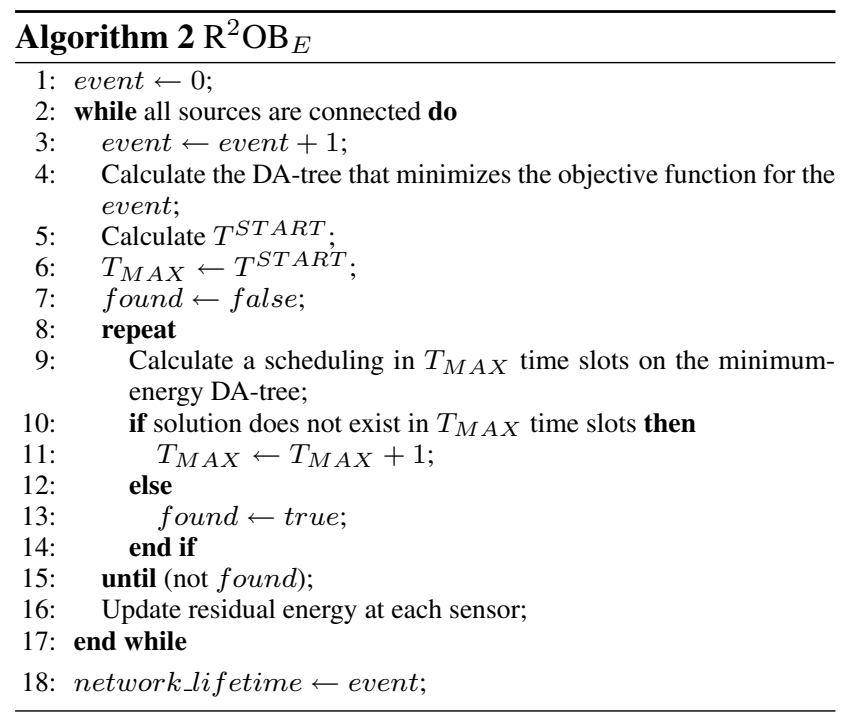

where $h$ is the distance in hops of the farthest source from the sink, and $T$ is such that

$$
|\mathcal{S}| \leq \sum_{i=0}^{T-1} 2^{i}
$$

The second member in (6) represents the maximum number of sources that can reach the sink in $T$ time slots. The DA-tree in $\mathrm{R}^{2} \mathrm{OB}_{L}$ is the solution of an ILP problem. Let us specify the formulation. Suppose that the residual energy of sensor $i$ is $E_{r e s}^{i}(n)$ at the $n$-th event. We calculate the mean $\bar{E}(n)$ of the residual energies at event $n$ :

$$
\bar{E}(n)=\frac{1}{N} \sum_{i=1}^{N} E_{\text {res }}^{i}(n)
$$

Then, for each link $(i, j)$ we compute $e_{i j}$ that is equal to the minimum between the residual energy at sensors $i$ and $j$ after the $(n+1)$-th event, if link $(i, j)$ is used to relay data for the $(n+1)$-th event. Hence,

$$
e_{i j}(n+1)=\min \left(E_{\text {res }}^{i}(n)-E_{i j}^{\text {trans }}, E_{\text {res }}^{j}(n)-E_{i j}^{r e c}\right)
$$

Finally, we define the cost matrix for re-routing strategies, where the generic element $c_{i j}$ has the form

$$
c_{i j}=\left|e_{i j}(n+1)-\bar{E}(n)\right|
$$

that represents the distance of the residual energy after the $(n+1)$-th event, $e_{i j}(n+1)$, from the current mean residual energy of the network $\bar{E}(n)$.

We represent the scheduling with a set of matrices $F^{t}$, one for each time slot $\left(1 \leq t \leq T_{M A X}\right)$. The element $f_{i j}^{t}$ in $F^{t}$ equals 1 if and only if a transmission occurs on link $(i, j)$ during time slot $t$. With the definitions above, along with those in Section 2, we can formulate the ILP as follows.

$$
\begin{aligned}
& \text { Minimize } \\
& c_{\text {tot }}=\sum_{t \in \mathcal{T}} \sum_{i \in \mathcal{N}} \sum_{j \in C_{i}} f_{i j}^{t} \cdot c_{i j} \\
& \text { with the following conditions: } \\
& \sum_{t \in \mathcal{T}} \sum_{j \in C_{i}} f_{i j}^{t}=1, \forall i \in \mathcal{S} \\
& 1 \leq \sum_{t \in \mathcal{T}} \sum_{i: O \in C_{i}} f_{i O}^{t} \leq|\mathcal{S}| \\
& \sum_{t \in \mathcal{T}} \sum_{i: O \in C_{i}} f_{i O}^{t}=0 \\
& \sum_{j \in C_{i}} f_{i j}^{t} \leq \sum_{\tau=1}^{t-1} \sum_{k: i \in C_{k}} f_{k i}^{\tau}, \forall t \in \mathcal{T}: t>1, \forall i \in \mathcal{N} \backslash\{\mathcal{S} \cup O\} \\
& \sum_{j: i \in C_{j}} f_{j i}^{t} \leq \sum_{\tau=t+1}^{T_{M A X}} \sum_{k \in C_{i}} f_{i k}^{\tau}, \forall t \in \mathcal{T}: t<T_{M A X}, \forall i \in \mathcal{N} \backslash O \\
& \sum_{j \in C_{i}} f_{i j}^{1}=0, \forall i \in \mathcal{N} \backslash \mathcal{S} \\
& \sum_{i: j \in C_{i}} f_{i j}^{T_{M A X}}=0, \forall j \in \mathcal{N} \backslash O \\
& \sum_{j \in C_{i}}\left(f_{i j}^{t}+f_{j i}^{t}\right) \leq 1, \forall t \in \mathcal{T}, \forall i \in \mathcal{N} \\
& N \cdot \sum_{j \in C_{i}} f_{i j}^{t}+\sum_{k \in C_{i}} \sum_{m: k \in C_{m}} f_{m k}^{t} \leq N, \forall i \in \mathcal{N}, \forall t \in \mathcal{T}, m \neq i
\end{aligned}
$$

Equation (10) is the objective function of the problem, and represents the total cost of the network for the event. This is obtained by weighing all transmissions, represented by the elements in $F^{t}$, for each $t \leq T_{M A X}$, with the cost associated with a transmission on that link. Remember that the cost matrix measures the gap between the forecasted residual energy at sensors and the current mean residual energy of the network, so as to achieve energy balancing. Note that the DA-tree can be seen as a union of flows, each departing from a source sensor and converging to the sink. A flow is the set of transmissions from a source to the sink. Constraints (11), (12), (13), (14) and (15) express conservation of flows. In particular, constraint (11) imposes that each source transmit only once. Constraint (12) imposes that the sink receive at least one and no more than $|\mathcal{S}|$ flows, while constraint (13) requires that the sink do not generate a flow. Constraints (14) and (15) are related to relay sensors. If a relay sensor transmits a packet in a time slot $t^{*} \geq 1$, it must have previously received the packet from another sensor in a time slot $t \leq t^{*}$. Constraint (16) allows only source sensors to transmit during the first time slot and constraint (17) imposes that the sink receives the information during the last time slot. The MAC constraints are imposed by (18) 


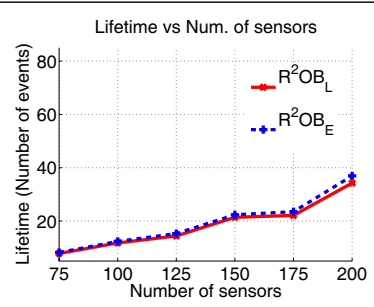

(a) Lifetime

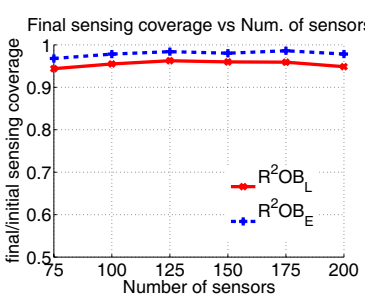

(b) Sensing Coverage Ratio

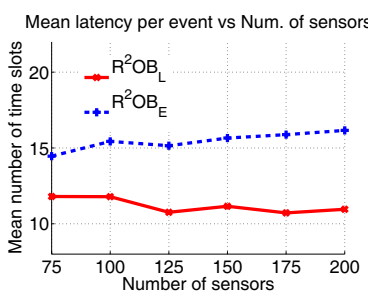

(c) Mean Latency per-event

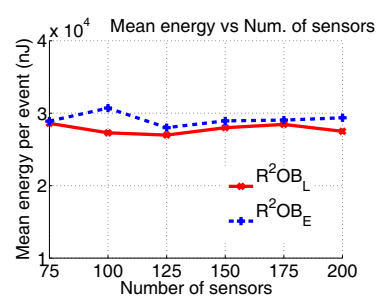

(d) Mean Energy per-event

Figure 4. Performance analysis of $R^{2} O B_{L}$ and $R^{2} O B_{E}$ as a function of the number of sensors

and (19). According to (18), each sensor can transmit to or receive from only one sensor in each time-slot. Constraint (19) is introduced to account for a collision-free MAC protocol. To this aim we impose that when a sensor $i$ is transmitting to a sensor $j$, no other sensors in the transmission range of $j$ can transmit, as it would collide with the reception of $j$. Similarly, $\mathrm{R}^{2} \mathrm{OB}_{E}$ finds the sequence of optimal DA-trees for each event. Two different ILPs are solved in each iteration of $\mathrm{R}^{2} \mathrm{OB}_{E}$. The first one computes the DAtree that minimizes the objective function, and consists of equations (10)-(13). The second one finds a scheduling for the transmissions on the DA-tree and consists of the remaining constraints; the tree computed in Step 1 of $\mathrm{R}^{2} \mathrm{OB}_{E}$ is an input parameter of the second problem.

\subsection{Analysis of the results}

In Figs. 4(a), 4(b), 4(c), and 4(d) we show the output of different optimization runs for $\mathrm{R}^{2} \mathrm{OB}_{L}$ and $\mathrm{R}^{2} \mathrm{OB}_{E}$. The considered area is a $30 \mathrm{~m} \times 30 \mathrm{~m}$ square, and $T_{R}=5 \mathrm{~m}$. The sink is positioned in the upper right corner, while 5 sources are placed in the lower left corner. In Fig. 4(a), the lifetime of the network is depicted with varying number of sensors, from 75 to 200 . The lifetime yielded by the two approaches is very similar, even if $\mathrm{R}^{2} \mathrm{OB}_{E}$ approach achieves better performance. Figure 4(b) compares the normalized final sensing coverage. We assume that each sensor can monitor a circular area around it with range smaller than $T_{R}$ (in

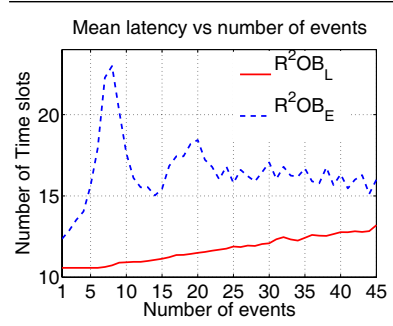

(a) Latency

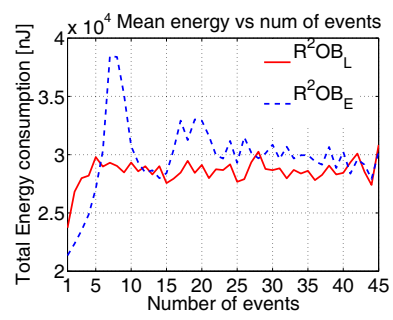

(b) Energy
Figure 5. Latency and energy trends. our optimization runs we consider a sensing range of $2 \mathrm{~m}$ ). The union of all these areas constitutes the sensing coverage of the network. When a sensor runs out of energy, it cannot monitor its area, so the sensing coverage decreases during the network lifetime. At the end of each optimization run, we compute the final sensing coverage with respect to the initial sensing coverage. The sensing coverage is high for both approaches, but $\mathrm{R}^{2} \mathrm{OB}_{E}$ performs slightly better because of the first energy balancing optimization. On the other hand, in the $\mathrm{R}^{2} \mathrm{OB}_{L}$ a lower per event mean latency and mean total energy consumption are achieved by means of the first latency minimization (see Figs. 4(c) and 4(d) respectively). Figures 5(a) and 5(b) show the variation of the latency and of the energy consumption during the lifetime of the network, for $\mathrm{R}^{2} \mathrm{OB}_{L}$ and $\mathrm{R}^{2} \mathrm{OB}_{E}$ respectively. For both approaches, the energy consumption is not steady. Instead, it alternatively increases and decreases. This is the effect of the balancing achieved by distributing energy consumption over time and space. Initially, the chosen DA-tree is the one with minimum energy cost (because all sensors have the same energy) and it is located close to the line that joins the sources to the sink. In succeeding events, the trees become alternatively wider and closer to that line, in order to distribute the energy consumption over all the region (as can be seen in the DA-trees depicted in Fig. 6(b) and 6(a)). Also the latency of $\mathrm{R}^{2} \mathrm{OB}_{E}$ presents an "accordionlike" behavior. Indeed, the latency increases when trees gets

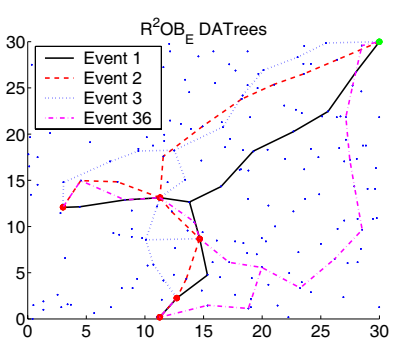

(a) $\mathrm{R}^{2} \mathrm{OB}_{E}$

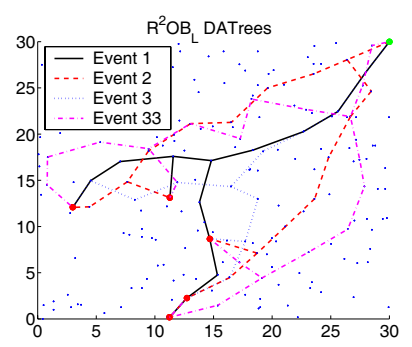

(b) $\mathrm{R}^{2} \mathrm{OB}_{L}$
Figure 6. Selection of DA-trees for $\mathbf{R}^{2} \mathbf{O B}_{x}$ 
wider and viceversa. On the other hand, the latency $\mathrm{R}^{2} \mathrm{OB}_{L}$ is monotonically increasing. This makes $\mathrm{R}^{2} \mathrm{OB}_{L}$ preferable to $\mathrm{R}^{2} \mathrm{OB}_{E}$. In fact, while in terms of energy the two approaches lead to similar results, $\mathrm{R}^{2} \mathrm{OB}_{L}$ assures a more predictable behavior and shorter time to complete events.

\section{Conclusion and future work}

In this paper we considered the problem of maximizing the lifetime of a WSN by combining data aggregation, re-routing and scheduling. We followed an incrementalcomplexity approach. First we considered a simple reference model where data flow routes do not change over time, multi-path routing is allowed and no data aggregation is in place. By means of LP optimization we have shown that in this scenario the energy consumption at all nodes can be perfectly equalized so as to maximize the network lifetime, with optimal routing patterns that involve two next-hops for each node - one close to the transmitter, the other closer to the sink.

Then, we extended further the model and considered dynamic re-routing and scheduling as means to balance the node energy consumption in time and maximize the network lifetime while maintaining a low data delivery latency. In fact, lifetime vs. latency emerges as the relevant trade-off dimension in the dynamic scenario. Two different re-routing algorithms were proposed and compared. We found that the $\mathrm{R}^{2} \mathrm{OB}_{L}$ algorithm displays better latency over $\mathrm{R}^{2} \mathrm{OB}_{E}$ with comparable performances regarding energy consumption and lifetime.

The proposed algorithms relay on a centralized view of the global network state. As a natural extension of this work, our future efforts will address distributed algorithms that can scale to large network size and automatically adapt to changes in the set of sources and to node mobility.

\section{References}

[1] I. F. Akyildiz, W. Su, Y. Sankarasubramaniam, and E. Cayirci, "Wireless sensor networks: A survey," Computer Networks (Elsevier), vol. 38, no. 4, pp. 393-422, Mar. 2002.

[2] J. Al-Karaki and A. Kamal, "Routing Techniques in Wireless Sensor Networks: a Survey," IEEE Wireless Communications, vol. 11, no. 6, pp. 6-28, December 2004.

[3] B. Krishnamachari, D. Estrin, and S. Wicker, "The impact of data aggregation in wireless sensor networks," in Proceedings of IEEE DEBS 2002, Vienna, Austria, July 2002.

[4] M. C. Vuran, O. B. Akan, and I. F. Akyildiz, "Spatiotemporal correlation: Theory and applications for wireless sensor networks," Computer Networks (Elsevier), vol. 45, no. 3, pp. 245-259, June 2004.

[5] J. H. Chang and L. Tassiulas, "Routing for maximum system lifetime in wireless ad-hoc networks," in Proc. of 37th Annual Allerton Conference on Communication, Control, and Computing, September 1997.
[6] — - "Energy conserving routing in wireless ad-hoc networks," in Proc. IEEE INFOCOM 2000, March 2000, pp. 22-31.

[7] — , "Fast approximation algorithms for maximum lifetime routing in wireless ad-hoc networks," in Proc. IEEE Networking 2000, vol. 1815, May 2000, pp. 702-713.

[8] N. Garg and J. Koenemann, "Faster and simpler algorithms for multicommodity flow and other fractional packing problems," in Proc. 39th Annual Symposium on Foundations of Computer Science, November 1998, pp. 300-309.

[9] A. Sankar and Z. Liu, "Maximum lifetime routing in wireless ad-hoc networks," in Proceedings of IEEE INFOCOM 2004, Hong Kong S.A.R., P.R. China, 2004.

[10] K. Kalpakis, K. Dasgupta, and P. Namjoshi, "Maximum lifetime data gathering and aggregation in wireless sensor networks," University of Maryland Technical Report, 2002.

[11] M. Bhardwaj and A. P. Chandrakasan, "Bounding the lifetime of sensor networks via optimal role assignments," in Proceedings of IEEE INFOCOM 2002, New York, NY, USA, 2002.

[12] Y. Yu, B. Krishnamachari, and V. K. Prasanna, "Energylatency tradeoffs for data gathering in wireless sensor networks," in Proceedings of IEEE INFOCOM 2004, Hong Kong S.A.R., P.R. China, 2004.

[13] R. K. Ahuja, T. L. Magnanti, and J. B. Orlin, Network Flows: Theory, Algorithms, and Applications. Englewood Cliffs, New Jersey: Prentice Hall, February 1993.

[14] M. R. Garey and D. S. Johnson, Computers and Intractability: A Guide to the Theory of NP-Completeness. New York, NY: W. H. Freeman \& Co., 1990.

[15] K. Kar, M. Kodialam, and T. Lakshman, "Minimum Interference Routing of Bandwidth Guaranteed Tunnels with MPLS Traffic Engineering Applications," IEEE Journal on Selected Areas in Communications, vol. 18, no. 12, December 2000.

[16] R. Fourer, D. M. Gay, and B. W. Kernighan, AMPL: A Modeling Language for Mathematical Programming. Duxbury Press / Brooks/Cole Publishing Company, 2002.

[17] CPLEX solver. [Online]. Available: http://www.cplex.com

[18] W. Heinzelman, A. Chandrakasan, and H. Balakrishnan, "An application-specific protocol architecture for wireless microsensor networks," IEEE Transactions on Wireless Communications, vol. 1, no. 4, pp. 660-670, Oct. 2002. 